\title{
Advances in Study on Structure of Polysaccharide
}

\author{
Miao $\mathrm{Yu}^{1,2, \mathrm{a}^{*}}$, Yue Cao ${ }^{1}$, Guosong Xin ${ }^{1,2}$, Chenfeng $\mathrm{Ji}^{1,2}$, Shiyong Gao ${ }^{1,2}$, \\ Xiang Zou ${ }^{1,2}$, Zheng Qi ${ }^{1,2}$, Yubin $\mathrm{Ji}^{1,2}$, Anna Qiao ${ }^{1}, \mathrm{Xin} \mathrm{Ru}^{1}$, Chi Wei ${ }^{1}$ \\ ${ }^{1}$ Center of Research and Development on Life Sciences and Environmental Sciences, \\ Harbin University of Commerce, Harbin, 150000, P.R. China \\ ${ }^{2}$ Engineering Research Center of Natural Anticancer Drugs, Ministry of Education, \\ Harbin University of Commerce, Harbin, 150076, P.R. China \\ a676247365@qq.com
}

Keywords: Polysaccharides, Structure, Structure analysis.

\begin{abstract}
Polysaccharide has many biological functions, such as anti-tumor, anti-virus and so on. The function of polysaccharide is closely related to its structure. The structure of polysaccharides can be classified into primary structure and advanced structure. There are many methods for the analysis of polysaccharide structure, which are mainly divided into chemical analysis method, instrumental analysis method, biological method, two-dimensional NMR spectrum and atomic force microscope method. The research progress on the relationship between the structure, structure and function of polysaccharide is reviewed.
\end{abstract}

\section{Introduction}

Polysaccharide is a kind of biological macromolecules formed by 10 or more sugars, which are formed by the linkage of the glycosides. It is one of the 4 basic substances of the biological organism. As an important bioactive substance, polysaccharide has many functions, such as immune regulation, anti-tumor, anti coagulation, reducing blood sugar and so on, and has been widely used in the fields of food, medicine, cosmetics and so on [1-4]. Many studies have indicated that the important role of polysaccharides is determined by its special structure. The relationship between the biological activity of polysaccharide and its advanced structure is more closely. So the research on polysaccharide structure has become a hot research topic, the research of polysaccharide structure provides a scientific basis for the development of new drugs and health products.

\section{Polysaccharide Structure}

The structure of polysaccharide is divided into primary structure and advanced structure, the primary structure is the primary structure, the two or three and the four level structures are the advanced structure. Polysaccharide structure is the composition of the sugar group, the arrangement order of the sugar group, the connection way of the adjacent sugar group, the different carbon structure and the sugar chain, the position and the length of the branch. The two stage structure of the polysaccharide is a regular conformation formed by the hydrogen bond between the main chain of the polysaccharide. The three stage structure and the four stage structure of the polysaccharide are based on the structure of the two stage, because of the non covalent interaction between the sugar units, resulting in the two level structure in the ordered space generated by the regular conformation.

\section{Analysis of the primary structure of polysaccharide}

Chemistry Analysis. (1) methylation: Methylation analysis is generally the reaction of all free hydroxyl groups of sugars to produce methyl ether. Then hydrolysis and release of methylated sugars. Then the NaBH4 is reduced to a sugar alcohol, and then the hydroxyl group is formed by acetylation and hydrolysis to obtain the methylated sugar alcohol acetyl derivative. By HPLC or GC 
analysis, determine the composition of the various types of sugar chain and the proportion of the various types of sugars, the connection position of the simple sugars, that is, the location of the glycosides.

(2) Periodate oxidation: Periodate can selectively fracture sugar molecules even dihydroxy or trihydroxy, generate the corresponding polysaccharide aldehyde, formaldehyde or formic acid. The quantitative reaction, each cracking a $\mathrm{C}$ - $\mathrm{C}$ key molecular periodate consumption. The release amount of periodate consumption and formic acid, can determine the key type and proportion of the glycosidic bond.

(3) Smith degradation: Smith is the degradation of polyhydroxy compounds periodate oxidation products reduced stable after partial acid hydrolysis. The hydrolysis products were identified by GC or HPLC, and the connection order and bond type of the sugars in the polysaccharide could be inferred by the product of the hydrolysis.

(4) Acid hydrolysis: acid hydrolysis to identify the composition of sugars in polysaccharides.

Instrument Analysis. (1) Ultraviolet spectrum (UV) [5]: UV spectra for polysaccharide detection at $260 \mathrm{~nm}$ and $280 \mathrm{~nm}$ with no characteristic absorption peak and to determine whether they contain nucleic acid or protein conjugates.

(2) Infrared spectroscopy (IR)[6]: Polysaccharide is often characterized by the characteristic absorption peak. $\alpha$-glycosidic link can be distinguished by $840 \mathrm{~cm}-1$ absorption peak. $\beta$-glycosidic link can be distinguished by $890 \mathrm{~cm}-1$ absorption peak. Pyranosides absorption peak at 1100 $1010 \mathrm{~cm}-1$ should be 3 strong. There are only 2 peaks in the corresponding region of furanoside. In addition, $810,870 \mathrm{~cm}-1$ is the mannose absorption peak, 1260 and $1730 \mathrm{~cm} \mathrm{-1}$ is the characteristic absorption peak of ester group or O- acetyl group. And esters in $1740 \mathrm{~cm}-1$, carboxylic acid ions in the $1600 \mathrm{~cm}-1,1414 \mathrm{~cm}-1$, amide in the $1650 \mathrm{~cm}-1,1550 \mathrm{~cm}-1$ near the absorption of vibration.

(3) Gas chromatography (GC) and High Performance Liquid Chromatography (HPLC): It is use for separation and identification of monosaccharide and methylated monosaccharide. PC HPG method using gel column in aqueous phase can also be used to determine the molecular weight of the sample. For GC, the need for sample derivatization, and HPLC can be directly into the sample. The GC sample can be made of sugar alcohol acetate derivatives, or sugar nitrile acetylation $[8,9]$.

(4) Nuclear magnetic resonance spectroscopy [10,11]: One of the major characteristics of the study of the structure of the sugar chain is not to destroy the samples by nuclear magnetic resonance spectroscopy. Nuclear magnetic resonance spectroscopy is currently used in the study of the structure of polysaccharide structure with 1HNMR and 13CNMR.1HNMR is mainly to solve the problem of the structure of the polysaccharide in the structure of glycosidic link. And 13C NMR chemical shifts of wide, good resolution, not only can help to identify the location of the various carbon, but also help distinguish the type and conformation of molecules, but also can use the relative peak height of the quantitative determination of the proportion of different residues in the polysaccharide.

(5) Electrophoresis technology: (1) Capillary electrophoresis [12] (CE): The electrophoresis technology is not only a separation means, can also be used for the oligosaccharide composition analysis, purity identification and ownership structure, and of oligosaccharide enzymatic hydrolysate with the method of qualitative analysis and quantitative analysis, in order to get the complete structure of the oligosaccharide chain. But except that the minority with carboxyl and sulfonate groups of the carbohydrate compounds, the vast majority of carbohydrate compounds without charge, very great, and there is no chromophore or fluorophore, so for the general high performance capillary electrophoresis (HPCE), adopts the following methods produce better effect: Such as derivatization to bring color and fluorophore or charge; and borate and other complex; and buffer additives form inclusion complexes; high $\mathrm{p} \mathrm{h}$ buffer conditions make the ionization; adding the surface active agent to form micelles. (2) Polyacrylamide gel electrophoresis (P-AGEFS) of fluorescent group labeled sugar: This is a simple and convenient method for structural analysis of oligosaccharides. Through to the molecules of carbohydrate of the reducing end carbonyl of fluorophores derived markers after polyacrylamide gel electrophoresis. This will not only enhance the detection sensitivity of the sugar molecules, and is usually in the form of carbohydrate 
molecules with neutral charge in the electrophoresis system were separated, can also be used to on multiple samples in parallel for the separation and qualitative analysis. (3) Twelve sodium dodecyl sulfate polyacrylamide gel electrophoresis (PAGE- PVDF SDS-): In this technique, the proteins on the gel electrophoresis were essentially transferred to polyvinyl fluoride (PVDF) film, which can be directly hydrolyzed by acid, and then the amino acid and carbohydrate compositions were analyzed. Hydrogen bromide degradation on this film, proteases, glycopeptidase and glycosidase hydrolysis, and PVDF membrane is directly arranged on the GC protein sequencer or MS sequence analysis was performed to obtain Pmol glycoprotein peptide and carbohydrate sequence and glycopeptide connection mode [13-15].

Biological Method. (1) Methods in Enzymology: Enzyme catalyzed reaction is highly specific and has less side products. It is an important method for structural analysis of sugar chains. Catalytic reaction of a polysaccharide substrate using alpha - and beta - glycosides, Can be used to confirm the type of the glycosides in the polysaccharide chain. Also available enzyme method to analyze the connection of glycopeptide.

(2) Immunological methods: Antigen and antibody will be combined with each other, a certain structure of polysaccharide (oligosaccharides) will inhibit the binding of antigen antibody, different sugar (Ban Kangyuan) have different inhibition constants. When some unknown structure of sugar chain against the combination of antigen and antibody produced an inhibitory, through determination of the inhibition constant and known structure of sugar chain compared, a similar inhibition constants of polysaccharide, its structure is similar, it is to use the immunoassay principle analysis of sugar chain structure.

Table 1. Analysis of the primary structure of polysaccharide.

\begin{tabular}{lll}
\hline Chemistry Analysis & Instrument Analysis & Biological Method \\
\hline methylation & UV & Methods in Enzymology \\
Periodate oxidation & IR & Immunological methods \\
Smith degradation & GC and HPLC & \\
Acid hydrolysis & Nuclear magnetic resonance & \\
& spectroscopy & \\
& Electrophoresis technology & \\
\hline
\end{tabular}

\section{Analysis of the Advanced Structure of Polysaccharide}

Two Dimensional NMR Spectrum. 2DNMR spectrum according to different needs can have many kinds, generally used there are two categories. D J decomposition spectra and two-dimensional chemical shift correlation spectroscopy (COSY). The general idea of polysaccharide structure: the 13CNMR spectra determine the anomeric carbon number, then according to the displacement of the anomeric carbon, HSQC (hydrogen and carbon short-range correlation) spectrum through cross peaks identify the anomeric protons, from the anomeric carbon of, in the COSY- 45 or DQF- cosy spectrum, through cross peaks identify the chemical shifts of the other hydrogen nuclei with 2D NMR analysis. If you can not fully identify, can be combined with COSY TOCSY, TQF-, NOESY and other help to identify. The displacement of carbon in the HSQC spectrum is found by the displacement of the hydrogen nucleus. For individual still unable to identify carbon, can be combined with the analysis of the composition of sugars and methylation results were speculated. Determine the main carbon and hydrogen, and then find the adjacent sugar units from the HMBC spectrum of the connection between the different carbon and different protons, so as to speculate the connection order. Jian hua Ju et al [16].using 2dnmr (H H cosy, H H TOCSY, HMQC, HMQCTOCSY and HMBC) in combination with chemical methods and IR, ESI MS identified from iron broken Gong roots of $\mathrm{n}$-butanol extract isolated a saponin of oleanolic acid 3 -o - $\beta$ D-glucopyranose $(1,3)-\alpha-$ L - THP nurse rhamnose $(1,2)-\alpha-L$ - pyran arabinose - 28 - O - $\alpha$ - L - pyran were rhamnose $(1-4) \beta$ - D-glucopyranose $(1,6)$ of the $\beta$ - D-glucopyranose glycosides.

Atomic Force Microscopy. Atomic force microscope (AFM) observation has the characteristics of 
high resolution and visualization [17]. AFM can be used to observe the morphology, the gel network and the subcellular structure of the polysaccharide, and the sample preparation is simple. It can detect the dynamic structure information of biological molecules under physiological conditions. AFM has two different modes of operation: namely, the contact mode (contact mode) and tapping mode (tapping mode), tapping mode in the scanning process of micro cantilever is oscillating tip continuous contact with the sample, which for the soft and difficult to be fixed biological materials (such as polysaccharides) has greater significance. Guang run Sun et al. [18]. Using AFM for the first time to see the micro structure of the licorice polysaccharide, is a pitch of six nm 2.5 fold helix, parallel array, spiral per share is $15 \sim 20 \mathrm{~nm}$. In addition, a new type of experimental technique based on AFM is a powerful tool to study the spatial structure of biological macromolecules such as polysaccharides.

Differential Scanning Calorimetry. In recent years it is in differential thermal analysis technology (thermal analysis differential, DTA) based on the development of a method can be used for the analysis of the advanced structure of polysaccharides [19]. It can not only describe the sample and reference than the temperature, real-time record heat difference variation with temperature, commonly used in evaluation of polymer thermal stability and compatibility and study of polymer material between the interaction [20,21]. At a given temperature, each system tends to achieve free energy minimum state, so in the process of heating or cooling. The sample was into structure state with different free energy, enthalpy change in the process of analysis, we can get some information of structural changes in the sample.

However, the application of DSC technology is still in the initial stage in the research field of the advanced structure of polysaccharide. Liu et al. [22]. Using the method of Ganoderma lucidum polysaccharide GLP20 (ganoderm a lucidum polysaccharide 20) conformation transition process were studied. As a result, in the DSC heating curve, endothermic peaks show intermolecular hydrogen bond breaking, GLP20 changed from triple helix structure is a single irregular coiled conformation, and after cooling reheating process, endothermic peak with the increase in the number of scans becomes small gradually, show that more than 135 DEG C heating caused by the conformational transition is irreversible. Differential scanning calorimetry (DSC) can also be senior structure of composite material, such as the application of the technique to study indicated that with the changes in the environment, carrageenan will conformational transition from helical to random coil form, and combined with macromolecular substances, due to the presence of steric hindrance, it cannot occur normal folding [23]. As a result, the DSC curve is larger endothermic peak as the evaluation of a drug delivery system, carrageenan and poly - (dimethylamino) phosphazene compound is stable is an important index [24].

$\mathrm{X}$ ray Diffraction (XRD). When $\mathrm{X}$ - ray diffraction analysis of a substance (usually a crystal), the material will produce different degrees of diffraction. Material composition, crystal form, intramolecular bonding method, molecular configuration, conformation and so on, determine the specific diffraction pattern of the material. For the polysaccharide, it can also be speculated that its symmetry, pitch and other helical parameters. It should be noted that the $\mathrm{X}$ ray diffraction of the sample must be highly ordered, at least to have enough of the crystallites. Because the vast majority of polysaccharide were not able to be crystallized, so before buying sugar samples X-ray diffraction must be after the induction, the simplest way is polysaccharide solution was placed on the fiber dilator, let it under the control of the humidity drying can be. Another way of $\mathrm{X}$ - ray diffraction, $\mathrm{X}$ ray fiber diffraction, shows an increasing application space in this area. At present, the combination of $\mathrm{X}$ - ray fiber diffraction method and computer simulation technology can be made to analyze the structure of Polysaccharide from atomic level.

Circular Dichroism (CD) [25-27]. Chiral molecules have optical activity. When the monochromatic left and right circularly polarized light is absorbed by a single sample, the sample is different from the left and right circular polarized light, which is called circle two color. The difference between the $\Delta \mathrm{A}=\Delta \mathrm{AL}-\Delta \mathrm{AR}$ known as Circular Dichroism values by wavelength scanning, signal amplification was Circular Dichroism (CD spectrum).If the external magnetic field effect, the CD signal can be further increased by several orders of magnitude, that is, the magnetic 
Circular Dichroism (MCD spectrum).Circular Dichroism has become an indispensable tool in the analysis of the spatial structure of biological macromolecules such as carbohydrates, proteins and nucleic acids. Gaio et al. [28]. Determination of the xanthan gum and mannan dextran mixtures in solution and colloidal $\mathrm{CD}$, data processing results show that the xanthan gum side chains participate in the gelling process. However, the $\mathrm{Z} n-3 \mathrm{P}$ quadrant rule is the basis of the microscopic theory of the determination of the conformation of carbohydrate compounds, and the general nature of this rule of thumb has yet to be confirmed. Also carbohydrate compounds CD database is still not perfect, and the chiral molecules and are researched with chromophores, thus limiting the scope of application of this technology.

\section{Conclusions}

UV, IR, GC, HPLC and CE are the main methods to study the structure of polysaccharides, and the main structural characteristics of polysaccharide molecules under different conditions can be comprehensively evaluated from various angles. And the advantages of atomic force microscopy (AFM) was mainly reflected in the visual and intuitive, CD and DSC technique is more used to describe different environment polysaccharide molecule structure change process, as for the NMR, mainly used as auxiliary means of analysis. In fact, the polysaccharide structure analysis method application in other areas of proteins, nucleic acids and other biological macromolecules have been more mature, and with polysaccharide to the complexity of the structure itself, resulting in there is still no a method should be used alone in the comprehensive analysis of polysaccharide advanced structure. Therefore, many kinds of analysis methods combined, can yet be regarded as polysaccharide advanced structure analysis of sound strategy, can improve the accuracy of the characterization of the structure of polysaccharide.

\section{Acknowledgments}

This work is supported by National Natural Science Foundation of China (81274067); Postdoctoral foundation of China (2015M581467); Natural Science Foundation of Heilongjiang Province (D201138); Training Program for Young Innovative Talented Person of Heilongjiang Province (UNPYSCT-2015071); Applied Technology Research and Development Project of Harbin (2016RAXXJ064); Science and Technology Innovation Team Program in Higher Education Institutions of Heilongjiang Province (2014TD009); Harbin Special Foundation for Young Technological Innovative Talented Person (2013RFQXJ150).

\section{References}

[1] W. J. Zhang, Biochemical research technology of glycoconjugate (Second Edition), Zhejiang University Press, 1999.

[2] S. G. Li, D. G. Wang, W. Tian, et al. Characterization and antitumor activity of a polysaccharide from Hedysarum polybotrys Hand-Mazz, Carbohydr. Polym. 73(2) (2008) 344-350.

[3] Y. J. Liu, S. H. Wang, M. Zhang, Y. F. Hua, Dendrobium polysaccharides with anticancer and immunological activity of, Guangzhou Chem. Ind. 4210 (2014) 58-60+65.

[4] G. J. Li, Zhaoxin, Large yellow croaker crude polysaccharide anti mutation effect and on HCT-116 node colonic cancer cells in vitro anticancer effect, Mod. Food Sci. Technol. 30(18008) (2014) 19-24.

[5] S. H. Lu, X. G. Yang, K. Wang, Q. P. Bell, Escherichia coli lipopolysaccharide UV detection method, Int. J. Med. Microbiol. 3221 (2011) 2466-2468+2471.

[6] Y. Diao, T. Liu, H. B. Han, Different regions of Auricularia polysaccharide and infrared spectroscopy study on the antioxidant activity of Hubei agricultural sciences, 55(55304) (2016) 
984-987+996.

[7] C. Q. Zhao, Study on the structure of polysaccharides and oligosaccharides from the capsule of the capsular reef, Ocean University of China, 2014.

[8] F. Q. Wang, Structural analysis of Polysaccharide from Z0206 and its effect on glucose and lipid metabolism in mice, Zhejiang University, 2013.

[9] L. Z. Lin, M. Jiao, Structural characterization and antioxidant activity of Neutral Polysaccharides from the leaves of the fruit of the light, Mod. Food Sci. Tech. 32 (2016) 106-110.

[10]D. Y. Liu, Y. R. Wang, H. F. Wei, R. Xia, X. Y. Yang, Fungicide cyflufenamid ghmbc spectral data, J. spectrosc. 3301 (2016) 142-152.

[11]X. J. Du, J. S. Zhang, Y. J. Pan, The role of nuclear magnetic resonance technology in the analysis of the structure of edible fungus polysaccharide, Ed. fungi in china, 29(16201) (2010) $3-6+19$.

[12]W. R. Cao, S. G. Zhang, X. J. Wang, Q. Q. Zhou, W. Zou, Advances in application of capillary electrophoresis in the analysis of protein biological products, Chem. Biol. Eng. 29(18506) (2012) 20-23.

[13]B. Zhang, Isatis monosaccharide extraction, degradation and capillary zone electrophoresis determination of, Hebei University, 2010.

[14]W. Tong, Construction and application of high performance capillary electrophoresis method for the separation and analysis of natural sugars, The Fourth Military Medical University, 2012.

[15]L. T. Chang, Dynamic coating modification of PDMS microfluidic chip and its application in the separation of oligosaccharides, Shaanxi Normal University, 2013.

[16]B. Slavomir, C. S. Shousun, et al. circular dichroism of the o- specific polysaccharide of vibrio cholerae o1 and some related derivatives, Carbohydr. Res. 270(2) (1995) 115- 122

[17]W. G. Xie, K. Q. Chen, Atomic force microscope probe selection and common trouble shooting method, Lab. Res. Explor. 33(22107) (2014) 19-22+52.

[18]J. Zhang, R. G. Sun, Atomic force microscope study on the spiral structure of the polysaccharide from the root of the root of, J. chem. 24 (2006) 2467-2472.

[19]M. B. Taouti, Y. Suffren, O. Leynaud, et al. Structures, thermal behaviors, and luminescent properties of anhydrous lanthanum iodatepolymorphs, Inorg. Chem. 54(7) (2015) 3608-3618.

[20]D. Suvakanta, M. P. Narsimha, D. Pulak, et al. Optimization and characterization of purified polysaccharide from Musa sapientum L. as apharmaceutical excipient, Food Chem. 149(8) (2014) 76-83.

[21]H. R. Kelidari, M. Saeedi, J. Akbari, et al. Formulation optimization and in vitro skin penetration of spironolactone loaded solid lipid nanoparticles, Colloids Surf B Biointerfaces, 128 (2015) 473-479.

[22] Y. Liu, J. Zhan, Q. Tang, et al. Physicochemical characterization of a high molecular weight bioactive $\beta$-D-glucan from the fruiting bodies of Ganoderma lucidum, Carbohyd. Polym. 101 (2014) 968-974.

[23] M. Yang, Y. Wu, J. Li, et al. Binding of curcumin with bovine serum albumin in the presence of l-carrageenan and implications on the stability and antioxidant activity of curcumin, J. Agric. Food Chem. 61(29) (2013) 7150-7155.

[24] V. Y. Grinberg, T. V. Burova, N. V. Grinberg, et al. Conformational energetic of interpolyelectrolyte complexation between 1-carrageenan and poly(methylaminophosphazene) measured by high-sensitivity differential scanning calorimetry, Langmuir, 27(12) (2011) 7714-7721. 
[25]R. E. Allan, et al. Induced circular dichroism study of the aqueous solution complexation of cello-oligosaccharides and related polysaccharides with aromatic dyes, Carbohydr. Res. 265(2) (1994) 181-195.

[26]Q. Shen, B. Huang, et al. Study on the interaction mechanism between enzyme and compound by Circular Dichroism, J. Zhongshan Univ. (Natural Science Edition), 45(4) (2006) 62-64.

[27]Z. Q. Yao, secondary gerile, etc. The effect of electric field on the two stage structure of lipase was studied by Circular Dichroism, Spectrosc. Spectral Anal. 26(12) (2006) 2311-2314.

[28]T. Gaio, C. Ester, B. Alberto, et al. Xanthan and glucomannan mixtures: synergistic interactions and gelation, Bioma-cromo-lecules, 3(3) (2002) 498-504. 